\title{
Distance Measures for Image Segmentation Evaluation
}

\author{
Xiaoyi Jiang, ${ }^{1}$ Cyril Marti, ${ }^{2}$ Christophe Irniger, ${ }^{2}$ and Horst Bunke ${ }^{2}$ \\ ${ }^{1}$ Computer Vision and Pattern Recognition Group, Department of Computer Science, University of Münster, Einsteinstrasse 62, \\ D-48149 Münster, Germany \\ ${ }^{2}$ Institute of Computer Science and Applied Mathematics, University of Bern, Neubrückstrasse 10, CH-3012 Bern, Switzerland
}

Received 17 March 2005; Revised 10 July 2005; Accepted 31 July 2005

\begin{abstract}
The task considered in this paper is performance evaluation of region segmentation algorithms in the ground-truth-based paradigm. Given a machine segmentation and a ground-truth segmentation, performance measures are needed. We propose to consider the image segmentation problem as one of data clustering and, as a consequence, to use measures for comparing clusterings developed in statistics and machine learning. By doing so, we obtain a variety of performance measures which have not been used before in image processing. In particular, some of these measures have the highly desired property of being a metric. Experimental results are reported on both synthetic and real data to validate the measures and compare them with others.
\end{abstract}

Copyright (๐ 2006 Hindawi Publishing Corporation. All rights reserved.

\section{INTRODUCTION}

Image segmentation and recognition are central problems of image processing for which we do not yet have any general purpose solution approaching human-level competence. Recognition is basically a classification task and one can empirically estimate the recognition performance (probability of misclassification) by counting classification errors on a test set. Today, reporting recognition performance on large data sets is a well-accepted standard. In contrast, segmentation performance evaluation remains subjective. Typically, results on a few images are shown and the authors argue why they look good. The readers frequently do not know whether the results have been opportunistically selected or are typical examples, and how well the demonstrated performance extrapolates to larger sets of images.

The main challenge is that the question "to what extent is this segmentation correct" is much more subtle than "is this face from person x." While a huge number of segmentation algorithms have been reported, there is only little work on methodologies of segmentation performance evaluation [1]. Several segmentation tasks can be identified: edge detection, region segmentation, and detection of curvilinear structures. Their performance evaluation is of quite different nature. For instance, an evaluation of detection algorithms for curvilinear structures must take the elongated shape of this particular feature into account [2]. In some sense, edge detection and region segmentation are two dual problems and their performance evaluation appears to be a similar task. One may convert a segmented region map to an equivalent edge map by marking the region boundaries only and then applying any edge detection evaluation method. However, a simple example, as shown in Figure 1, reveals a fundamental difference: although in terms of the boundaries the two segmentation results only differ marginally, their discrepancy in the number of regions is substantially larger. This latter aspect has not been a real concern in evaluating edge detectors [3]. For this reason, we need separate strategies for evaluating region segmentation algorithms.

In the present paper, we are concerned with region segmentation. Note that thresholding may be considered a special case of region segmentation (into two or more regions with unique semantic labels). The evaluation of thresholding techniques is a topic of its own right and the readers are referred to the recent survey paper [4].

The various methods for performance evaluation, in general, can be categorized according to the following taxonomy [1]:

(i) theoretical evaluation,

(ii) experimental evaluation:

(a) feature-based evaluation:

(1) non-GT (ground-truth)-based evaluation;

(2) GT-based evaluation,

(b) task-based evaluation.

A theoretical evaluation is done by applying a mathematical analysis without the algorithms ever being implemented and applied to an image. Instead, the algorithm behavior is mathematically characterized and the performance is determined 


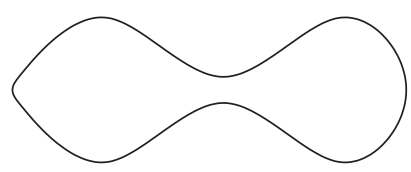

(a)

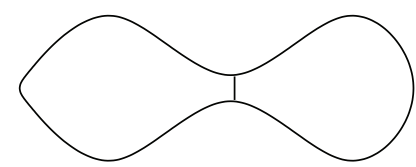

(b)

Figure 1: Two segmentation results.

analytically or by simulation. The major limitations of theoretical approaches are the simplistic mathematical models and the difficulty in applying them to many of the more modern segmentation algorithms because of their complexity. An experimental evaluation can be divided into featurebased and task-based. The former category measures the algorithm performance only based on the quality of detected features under consideration, for example, edges and regions. Within this category, we can further distinguish between non-GT-based and GT-based approaches. The basic idea of GT-based approaches is to measure the difference between the machine segmentation result and the ground truth (expected ideal segmentation, which is in almost all cases specified manually). In contrast, non-GT-based methods do not assume the availability of GT and compute performance measures directly by means of some desirable properties of the segmentation result. Task-based evaluation follows a very different philosophy. Image segmentation represents only one, although important, step in achieving the highlevel goal of a vision system, for example, object recognition. Of ultimate interest is the overall performance of the system. Instead of abstractly comparing the performance of segmentation algorithms, it may be thus more meaningful to conduct an indirect comparison based on their influences on the final performance of the entire system.

In this paper, we follow the GT-based evaluation paradigm. We propose to consider the image segmentation problem as one of data clustering and, as a consequence, to use measures for comparing clusterings developed in statistics and the machine learning community for the purpose of segmentation evaluation. This novel approach opens the door for a variety of measures which have not been used before in image processing. As we will see later, some of the measures even have the highly desired property of being a metric. Note that this paper is a substantially extended version of [5]. The extension includes a new distance measure based on bipartite graph matching, more detailed discussion of the distance measures and their properties, and additional comparison work (Sections 4 and 5.3).

The rest of the paper is structured as follows. We start with a short discussion of related work. Then, measures for comparing clusterings are presented, followed by their theoretical and experimental validations. Finally, some discussions conclude the paper.

\section{RELATED WORK}

In [6], a machine segmentation (MS) of an image is compared to the ground-truth specification to count instances of correct segmentation, under-segmentation, over-segmentation, missed regions, and noise regions. These measures are defined based on the degree of mutual overlap required between a region in MS and a region in GT. A correctly segmented region is recorded if and only if an MS region and the corresponding GT region have a mutual overlap greater than a threshold $T$. Multiple MS regions that together correspond to one GT region constitute an instance of over-segmentation, while one MS region corresponding to the union of several GT regions is considered as undersegmentation. An MS (GT) region that has no corresponding in GT (MS) constitutes an instance of noise (missing) region. This evaluation method is widely used for texture segmentation [7] and range image segmentation [6, 8-11].

In contrast, the approach from [12] delivers one single performance measure. Considering two different segmentations $S_{1}=\left\{R_{1}^{1}, R_{1}^{2}, \ldots, R_{1}^{m}\right\}$ and $S_{2}=\left\{R_{2}^{1}, R_{2}^{2}, \ldots, R_{2}^{n}\right\}$ of the same image, we associate each region $R_{2}^{i}$ from $S_{2}$ with a region $R_{1}^{j}$ from $S_{1}$ such that $R_{2}^{i} \cap R_{1}^{j}$ is maximal. The directional Hamming distance from $S_{1}$ to $S_{2}$ is defined as

$$
D_{H}\left(S_{1} \Longrightarrow S_{2}\right)=\sum_{R_{2}^{i} \in S_{2}} \sum_{R_{1}^{k} \neq R_{1}^{j}}\left|R_{1}^{k} \cap R_{2}^{i}\right|
$$

corresponding to the total area under the intersections between all $R_{2}^{i} \in S_{2}$ and their nonmaximally intersected regions $R_{1}^{k}$ from $S_{1}$. The reversed distance $D_{H}\left(S_{2} \Rightarrow S_{1}\right)$ can be similarly computed. Finally, the overall performance measure is given by

$$
p=1-\frac{D_{H}\left(S_{1} \Longrightarrow S_{2}\right)+D_{H}\left(S_{2} \Longrightarrow S_{1}\right)}{2 A},
$$

where $A$ is the image size and $p \in[0,1]$. Letting MS and GT play the role of $S_{1}$ and $S_{2}$, respectively, allows us to measure their discrepancy. Recently, this index has been used to compare several segmentation algorithms by integration of region and boundary information [13].

In [14], another single overall performance measure is proposed. It is designed so that if one region segmentation is a refinement of another (at different granularities), then the measure should be small or even zero. Let $R\left(S, p_{i}\right)$ be the set of pixels corresponding to the region in segmentation $S$ that contains the pixel $p_{i}$. Then, the local refinement error associated with $p_{i}$ is

$$
E\left(S_{1}, S_{2}, p_{i}\right)=\frac{\left|R\left(S_{1}, p_{i}\right) \backslash R\left(S_{2}, p_{i}\right)\right|}{\left|R\left(S_{1}, p_{i}\right)\right|},
$$

where $\backslash$ denotes set difference. Finally, the overall performance measure is defined as

$$
\mathrm{GCE}=\frac{1}{A} \min \left\{\sum_{\text {all pixels } p_{i}} E\left(S_{1}, S_{2}, p_{i}\right), \sum_{\text {all pixels } p_{i}} E\left(S_{2}, S_{1}, p_{i}\right)\right\},
$$


or

$$
\mathrm{LCE}=\frac{1}{A} \sum_{\text {all pixels }} \min \left\{E\left(S_{i}, S_{2}, p_{i}\right), E\left(S_{2}, S_{1}, p_{i}\right)\right\},
$$

where GCE and LCE stand for global consistency and local consistency error, respectively. Note that both measures are tolerant of refinement. In the extreme case, a segmentation containing a single region and a segmentation consisting of regions of a single pixel are rated by $p_{1}=p_{2}=0$. Due to their tolerance of refinement, these two measures are not sensible to over- and under-segmentation and may be therefore not applicable in some evaluation situations.

\section{MEASURES FOR COMPARING CLUSTERINGS}

Given a set of objects $O=\left\{o_{1}, \ldots, o_{n}\right\}$, a clustering of $O$ is a set of subsets $C=\left\{c_{1}, \ldots, c_{k}\right\}$ such that $c_{i} \subseteq O, c_{i} \cap c_{j}=\varnothing$ if $i \neq j, \bigcup_{i=1}^{k} c_{i}=O$. Each $c_{i}$ is called a cluster. Clustering has been extensively studied in the statistics and machine learning community [15]. In particular, several measures have been proposed to quantify the difference between two clusterings $C_{1}=\left\{c_{11}, \ldots, c_{1 k}\right\}$ and $C_{2}=\left\{c_{21}, \ldots, c_{2 l}\right\}$ of the same set $O$.

If we interpret an image as a set $O$ of pixels and a segmentation as a clustering of $O$, then these measures can be applied to quantify the difference between two segmentations, for example, between MS and GT. This view of the segmentation evaluation tasks opens the door for a variety of measures which have not been used before in image processing. As we will see later, some of the measures are even metrics, being a highly desired property which is not fulfilled by the measures discussed in the last section. In the following, we present three classes of measures.

\subsection{Distance of clusterings by counting pairs}

Given two clusterings $C_{1}$ and $C_{2}$ of a set $O$ of objects, we consider all pairs of objects $\left(o_{i}, o_{j}\right), i \neq j$, from $O \times O$. A pair $\left(o_{i}, o_{j}\right)$ falls into one of the four categories:

(i) in the same cluster under both $C_{1}$ and $C_{2}$ (the total number of such pairs is represented by $\left.N_{11}\right)$,

(ii) in different clusters under both $C_{1}$ and $C_{2}\left(N_{00}\right)$,

(iii) in the same cluster under $C_{1}$ but not $C_{2}\left(N_{10}\right)$,

(iv) in the same cluster under $C_{2}$ but not $C_{1}\left(N_{01}\right)$.

Obviously, $N_{11}+N_{00}+N_{10}+N_{01}=n(n-1) / 2$ holds, where $n$ is the cardinality of $O$.

Several distance measures, also called indices, for comparing clusterings are based on these four counts. The Rand index introduced in [16] is defined as

$$
\mathcal{R}\left(C_{1}, C_{2}\right)=1-\frac{N_{11}+N_{00}}{n(n-1) / 2} .
$$

Note that the original definition was actually given by $1-$ $\mathcal{R}\left(C_{1}, C_{2}\right)$. The only difference is that the former is a distance (dissimilarity) while the latter is a similarity measure. For comparison purpose, we consistently use distance measures such that a value of zero implies a perfect matching, that is, two identical clusterings. This remark applies to the two indices below as well.

Fowlkes and Mallows [17] introduce the following index:

$$
\mathcal{F}\left(C_{1}, C_{2}\right)=1-\sqrt{W_{1}\left(C_{1}, C_{2}\right) W_{2}\left(C_{1}, C_{2}\right)}
$$

as the geometric mean of

$$
\begin{aligned}
& W_{1}\left(C_{1}, C_{2}\right)=\frac{N_{11}}{\sum_{i=1}^{k} n_{i}\left(n_{i}-1\right) / 2}, \\
& W_{2}\left(C_{1}, C_{2}\right)=\frac{N_{11}}{\sum_{j=1}^{l} n_{j}\left(n_{j}-1\right) / 2},
\end{aligned}
$$

where $n_{i}$ stands for the size of the $i$ th element of $C_{1}$ and $n_{j}$ the $j$ th element of $C_{2}$. The terms $W_{1}$ and $W_{2}$ represent the probability that a pair of points which are in the same cluster under $C_{1}$ are also in the same cluster under $C_{2}$ and vice versa.

Finally, the Jacard index [18] is given by

$$
\mathscr{g}\left(C_{1}, C_{2}\right)=1-\frac{N_{11}}{N_{11}+N_{10}+N_{01}} .
$$

It is easy to see that the three indices are all distance measures with a value domain $[0,1]$. The value is zero if and only if the two clusterings are the same except for possibly assigning different names to the individual clusters, or listing the clusters in different order. The case with value one corresponds to the maximum degree of cluster dissimilarity, for example, $C_{1}$ contains a single cluster while $C_{2}$ consists of clusters of a single object.

\subsection{Distance of clusterings by set matching}

This second class of comparison criteria is based on matching the clusters of two clusterings. The term

$$
a\left(C_{1}, C_{2}\right)=\sum_{c_{i} \in C_{1}} \max _{c_{j} \in C_{2}}\left|c_{i} \cap c_{j}\right|
$$

measures the matching degree between the clusters of $C_{1}$ and $C_{2}$ and takes the maximum value $n$ only if $C_{1}=C_{2}$. Similarly, a term $a\left(C_{2}, C_{1}\right)$ can be defined. Based on these two terms, van Dongen [19] proposes the index

$$
\mathscr{D}\left(C_{1}, C_{2}\right)=2 n-a\left(C_{1}, C_{2}\right)-a\left(C_{2}, C_{1}\right)
$$

and proves that it is a metric. This index is closely related to the performance measure $p$ in [12]. The only difference is that the former is a distance (dissimilarity) measure while the latter is a similarity measure and they can be mapped to each other by a simple linear transformation $\mathcal{D}\left(C_{1}, C_{2}\right)=$ $2 n(1-p)$.

Besides this index known from the literature, we propose in the following a novel procedure for measuring the distance of two clusterings based on bipartite graph matching. We represent the two given clusterings $C_{1}$ and $C_{2}$ as one common set of nodes $\left\{c_{11}, \ldots, c_{1 k}\right\} \cup\left\{c_{21}, \ldots, c_{2 l}\right\}$ of a graph, that is, each cluster from either $C_{1}$ or $C_{2}$ is regarded as a node. Then, an edge is inserted between each pair of nodes $\left(c_{1 i}, c_{2 j}\right)$. The 
weight of this edge is equal to $\left|c_{1 i} \cap c_{2 j}\right|$, that is, it is equal to the number of elements that occur in both $c_{1 i}$ and $c_{2 j}$.

Given this graph, we determine a maximum-weight bipartite graph matching. Such a matching is defined by a subset $\left\{\left(c_{1 i_{1}}, c_{2 j_{1}}\right), \ldots,\left(c_{1 i_{r}}, c_{2 j_{r}}\right)\right\}$ such that each of the nodes $c_{1 i}$ and $c_{2 j}$ has at most one incident edge, and the total sum of weights is maximized over all possible subsets of edges. Intuitively, the maximum-weight bipartite graph matching can be understood as a correspondence between the clusters of $C_{1}$ and the clusters of $C_{2}$ such that no two clusters of $C_{1}$ are mapped to the same cluster in $C_{2}$, and vice versa. Moreover, the correspondence optimizes the total number of objects that belong to corresponding clusters. Algorithms for computing maximum-weight bipartite graph matching can be found in [20], for example.

The sum of weights $w$ of a maximum-weight bipartite graph matching is bounded by the number of objects $n$ in set $O$. Therefore, a suitable normalized measure for the distance of $C_{1}$ and $C_{2}$ is

$$
\mathscr{B} g_{\mathcal{M}}\left(C_{1}, C_{2}\right)=1-\frac{w}{n}
$$

Clearly, this measure is equal to 0 if and only if $k=l$ and there is a bijective mapping $f$ between the clusters of $C_{1}$ and $C_{2}$, such that $c_{1 i}=f\left(c_{1 i}\right)$ for $i \in\{1, \ldots, k\}$. Values close to one indicate that no good mapping between the clusters of $C_{1}$ and $C_{2}$ exists, such that corresponding clusters have many elements in common.

\subsection{Information-theoretic distance of clusterings}

Mutual information (MI) is a well-known concept in information theory. It measures how much information about random variable $Y$ is obtained from observing random variable $X$. Let $X$ and $Y$ be two random variables with joint probability distribution $p(x, y)$ and marginal probability functions $p(x)$ and $p(y)$. Then, the mutual information of $X$ and $Y, \operatorname{MI}(X, Y)$, is defined as

$$
\operatorname{MI}(X, Y)=\sum_{(x, y)} p(x, y) \log \frac{p(x, y)}{p(x) p(y)} .
$$

Some properties of MI are summarized below; for a more detailed treatment, the reader is referred to [21],

(i) $\operatorname{MI}(X, Y)=\operatorname{MI}(Y, X)$.

(ii) $\operatorname{MI}(X, Y) \geq 0$.

(iii) $\operatorname{MI}(X, Y)=0$ if and only if $X$ and $Y$ are independent.

(iv)

$$
\operatorname{MI}(X, Y) \leq \min (H(X), H(Y)),
$$

where $H(X)=-\sum_{x} p(x) \log p(x)$ is the entropy of random variable $X$.

(v)

$$
\operatorname{MI}(X, Y)=H(X)+H(Y)-H(X, Y),
$$

where $H(X, Y)=-\sum_{(x, y)} p(x, y) \log p(x, y)$ is the joint entropy of $X$ and $Y$.
In the context of measuring the distance of two clusterings $C_{1}$ and $C_{2}$ over a set $O$ of objects, the discrete values of random variable $X$ are the different clusters $c_{i} \in C_{1}$ an element of $O$ can be assigned to. Similarly, the discrete values of $Y$ are the different clusters $c_{j} \in C_{2}$ an object of $O$ can be assigned to. Hence, the equation above becomes

$$
\operatorname{MI}\left(C_{1}, C_{2}\right)=\sum_{c_{i} \in C_{1}} \sum_{c_{j} \in C_{2}} p\left(c_{i}, c_{j}\right) \log \frac{p\left(c_{i}, c_{j}\right)}{p\left(c_{i}\right) p\left(c_{j}\right)} .
$$

As $\operatorname{MI}\left(C_{1}, C_{2}\right) \leq \min \left(H\left(C_{1}\right), H\left(C_{2}\right)\right)$ and $H(C) \leq \log k$, with $k$ being the number of clusters present in clustering $C$, the upper bound of $\operatorname{MI}\left(C_{1}, C_{2}\right)$ depends on the number of clusters in $C_{1}$ and $C_{2}$. To get a normalized value, it was proposed to divide $\operatorname{MI}(X, Y)$ by $\log (k \cdot l)$, where $k$ and $l$ are the numbers of discrete values of $X$ and $Y$, respectively [22]. This leads to the normalized mutual information

$$
\begin{aligned}
\mathcal{N} \mathcal{M} & \ell\left(C_{1}, C_{2}\right) \\
& =1-\frac{1}{\log (k \cdot l)} \sum_{c_{i} \in C_{1}} \sum_{c_{j} \in C_{2}} p\left(c_{i}, c_{j}\right) \log \frac{p\left(c_{i}, c_{j}\right)}{p\left(c_{i}\right) p\left(c_{j}\right)} .
\end{aligned}
$$

Meila [23] suggests a further alternative called variation of information:

$$
\mathcal{V} I\left(C_{1}, C_{2}\right)=H\left(C_{1}\right)+H\left(C_{2}\right)-2 \mathrm{MI}\left(C_{1}, C_{2}\right),
$$

where

$$
\begin{aligned}
& H\left(C_{1}\right)=-\sum_{c_{i} \in C_{1}} p\left(c_{i}\right) \log \left(c_{i}\right), \\
& H\left(C_{2}\right)=-\sum_{c_{j} \in C_{2}} p\left(c_{j}\right) \log \left(c_{j}\right)
\end{aligned}
$$

represent the entropy of $C_{1}$ and $C_{2}$, respectively. In general, this index is bounded by $\log n$, which is reached in the case when a cluster $C_{1}$ contains a single cluster and a cluster $C_{2}$ consists of clusters of a single object. If, however, $C_{1}$ and $C_{2}$ have at most $K, K \leq \sqrt{n}$, clusters each, the $\mathcal{V} I\left(C_{1}, C_{2}\right)$ is bounded by $2 \log K$. Importantly, the index turns out to be a metric.

\subsection{Remarks}

Among the seven distance measures introduced above, $\mathscr{D}\left(C_{1}, C_{2}\right)$ and $\mathcal{V} I\left(C_{1}, C_{2}\right)$ are provably metrics. The other measures satisfy all properties of a metric except the triangle inequality, for which we are not aware of any proof or counterexample. Note that a comparison criterion that is a metric has several advantages. Among others, it makes the criterion more understandable and matches the human intuition better than an arbitrary distance function of two variables.

At first glance, the distance measures given in Section 3.1 pose some efficiency problems. In fact, a naive approach to computing $N_{11}, N_{00}, N_{10}$, and $N_{01}$ would need $O\left(N^{4}\right)$ operations when dealing with images of size $N \times N$. Fortunately, we may make use of the confusion matrix, also called association 


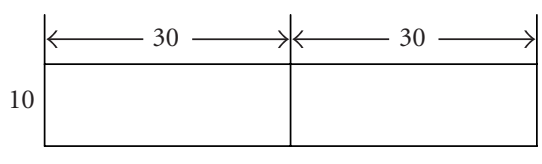

(a)

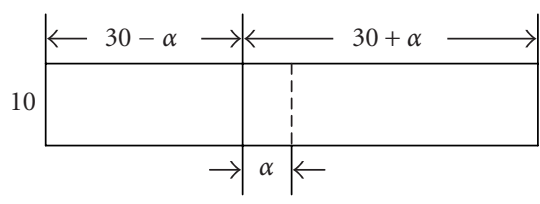

(b)

FIgURE 2: (a) GT and (b) MS of an image of size $10 \times 60$.

matrix or contingency table, of $C_{1}$ and $C_{2}$. It is a $k \times l$ matrix, whose $i j$ th element $m_{i j}$ represents the number of points in the intersection of $c_{i}$ of $C_{1}$ and $c_{j}$ of $C_{2}$, that is, $m_{i j}=\left|c_{i} \cap c_{j}\right|$. It can be shown (see the appendix) that

$$
\begin{aligned}
& N_{11}=\frac{1}{2}\left[\sum_{i=1}^{k} \sum_{j=1}^{l} m_{i j}^{2}-n\right] \\
& N_{00}=\frac{1}{2}\left[n^{2}-\sum_{i=1}^{k} n_{i}^{2}-\sum_{j=1}^{l} n_{j}^{2}+\sum_{i=1}^{k} \sum_{j=1}^{l} m_{i j}^{2}\right], \\
& N_{10}=\frac{1}{2}\left[\sum_{i=1}^{k} n_{i}^{2}-\sum_{i=1}^{k} \sum_{j=1}^{l} m_{i j}^{2}\right] \\
& N_{01}=\frac{1}{2}\left[\sum_{j=1}^{l} n_{j}^{2}-\sum_{i=1}^{k} \sum_{j=1}^{l} m_{i j}^{2}\right] .
\end{aligned}
$$

These relationships reduce the computational complexity to $O\left(N^{2}\right)$ only and thus make the indices presented in Section 3.1 tractable for large-scale clustering problems like image segmentation. Finally, it is noteworthy that all the other measures can be easily computed from the confusion matrix as well.

The computational complexity of the distances by counting pairs amounts to $O\left(N^{2}+k l\right)$. Since typically $k<N$ and $l<$ $N$ hold, we basically have a quadratic complexity $O\left(N^{2}\right)$. The same applies to the index $\mathscr{D}\left(C_{1}, C_{2}\right)$ and the informationtheoretic distances. Since the index $\mathcal{B} \mathscr{G} \mathcal{M}\left(C_{1}, C_{2}\right)$ only requires a maximum-weight bipartite graph matching, it can be computed in low polynomial time as well.

\section{COMPARISON WITH HOOVER INDEX}

In evaluating the measures defined in the last section, we did some comparison work. For this purpose, we consider the Hoover measure [6] and the measures from [14]. The measure from [12] was ignored because of its equivalence to the van Dongen index.

We first present some theoretical considerations related to the Hoover index before turning to experimental evaluation in the next section. Among the five performance measures from [6] only the correct detection $C D$ is used. A distance measure ( $1-C D / \# \mathrm{GT}$ regions $)$ is obtained for comparison purpose.

The Hoover index depends on the overlap threshold $T$. One may expect that it monotonically increases, that is, becomes worse, with increasing tolerance threshold T. However, this is not true. It may happen that the Hoover index becomes larger with increasing $T$ values. If we only choose a particular value of $T$, this kind of inconsistency may cause some unexpected effects in comparing different algorithms. ${ }^{1}$

Another inherent problem of the Hoover index is its insensitivity to distortion. Basically, this index counts the number of correctly detected regions. Increasing distortion level has no influence on the count at all as far as the tolerance threshold $T$ does not become effective. The simple example in Figure 2 illustrates this situation. In the machine segmentation, the region boundary is shifted to left by a distance $\alpha$. As far as $\alpha \leq 30(1-T)$, the Hoover index consistently indicates a perfect segmentation (consisting of two correct detected regions). The measures proposed in this paper, however, are all pixel-based. As such they sensitively react to the distortions.

\section{EXPERIMENTAL VALIDATION}

In the following, we present experiments to validate the proposed measures based on both synthetic and real data. The experiments were conducted in range image domain and intensity image domain.

\subsection{Validation on synthetic data}

The range image sets reported in $[6,11]$ have become popular for evaluating range image segmentation algorithms. Totally, three image sets with manually specified ground truth are available: ABW and Perceptron for planar surfaces and K2T for curved surfaces. ABW and K2T are structured light sensors, while Perceptron is a time-of-flight laser scanner. Each range image has a manually specified GT segmentation. Since range image segmentation is geometrically driven, the GT is basically unique and there is no need to work with multiple GT segmentations as is the case in dealing with intensity images (see Section 5.3). More details and a comparison of the three image sets can be found in [1]. For each GT image, we constructed several synthetic MS results in the following way. A point $p$ is selected randomly. We find the point $q$ nearest to $p$ which does not belong to the same region as $p$. Then, $q$ is switched to the region of $p$ provided that this step will not produce additional regions. This basic operation is repeated

\footnotetext{
${ }^{1}$ One possibility to alleviate the problem is to define a single performance measure based on multiple $T$ values. In [10], the authors use the area under the performance curve for this purpose, which corresponds to the average performance of an algorithm over a range of thresholds.
} 


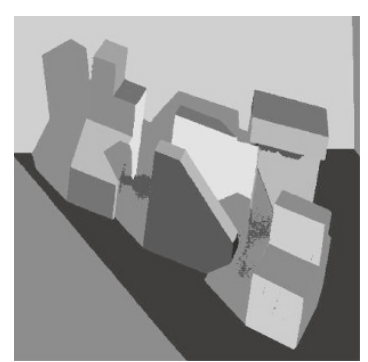

(a)

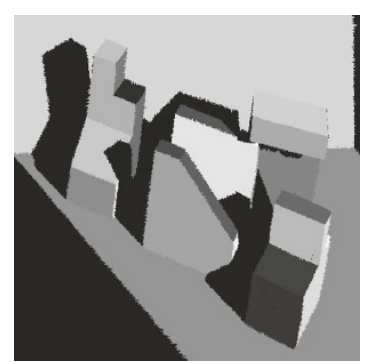

(b)

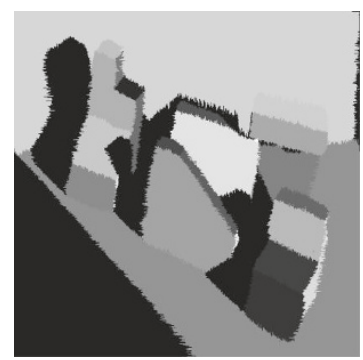

(c)

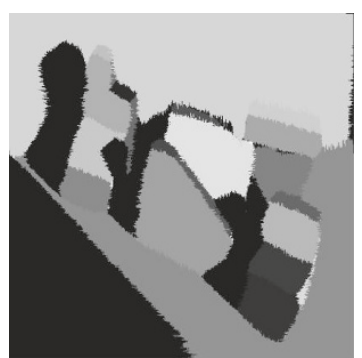

(d)

Figure 3: An ABW image: (a) GT, synthetic MS, (b) 5\% distortion, (c) 30\% distortion, (d) 50\% distortion.

TABLE 1: Hoover index for an ABW image. The two instances of inconsistency are underlined.

\begin{tabular}{lcllllllll}
\hline Distortion level & $T=0.55$ & 0.60 & 0.65 & 0.70 & 0.75 & 0.80 & 0.85 & 0.90 & 0.95 \\
\hline $10 \%$ & 0.222 & 0.333 & 0.333 & 0.444 & 0.556 & 0.556 & 0.556 & 0.667 & 0.889 \\
$20 \%$ & 0.778 & $\underline{0.667}$ & $\underline{0.667}$ & $\underline{0.667}$ & $\underline{0.667}$ & 0.778 & 0.778 & 0.778 & 1.000 \\
$30 \%$ & 0.778 & 0.778 & 0.778 & 0.889 & 0.889 & 0.889 & $\underline{0.778}$ & 0.889 & 1.000 \\
$40 \%$ & 0.889 & 1.000 & 1.000 & 1.000 & 1.000 & 1.000 & 1.000 & 1.000 & 1.000 \\
\hline
\end{tabular}

for some $d \%$ of all points. Figure 3 shows one of the ABW GT images and three generated MS versions.

The Hoover index does not necessarily monotonically increase, that is, becomes worse, with increasing tolerance threshold $T$. Table 1 lists the Hoover index for a particular ABW image as a function of $T$ and the distortion level $d$. There are two instances of inconsistencies. At distortion level $30 \%$, for example, the index value 0.778 for $T=0.85$ is lower than 0.889 for $T=0.80$. In addition, Table 1 also illustrates the insensitivity of the Hoover index to distortions. For $T=0.85$, for instance, the Hoover index remains unchanged (0.778) at both distortion levels 20\% and 30\%. Objectively, however, a significant difference is visible and should be reflected in the performance measures. Obviously, the Hoover index does not perform as one would expect here.

By definition, the indices introduced in this paper have a high sensitivity to distortions. Table 2 lists the average values for all thirty ABW test images. ${ }^{2}$ No inconsistencies occur here, and the values are strict monotonically increasing with a growing amount of distortion.

Experiments have also been conducted using the Perceptron image set, and we observed similar behavior of the indices. So far, the K2T image set was not tested yet, but we do not expect diverging outcome.

\subsection{Validation on real range images}

The Hoover index has been applied to evaluate a variety of range image segmentation algorithms $[6,8,9]$. In our experiments, we only considered the four algorithms compared in

\footnotetext{
2 The ABW image set contains forty images and is divided into ten training images and thirty test images. Only the test images were used in our experiments.
}

the original work [6]: University of Edinburgh (UE), University of Bern (UB), University of South Florida (USF), and University of Washington (UW). Table 3 reports an evaluation of these algorithms by means of the indices introduced in this paper. The results imply a ranking of segmentation quality: UE, UB, USF, UW, which coincides well with the ranking from the Hoover index (compare the Hoover index values for $T=0.85$ in Table 3 and the original work [6]). Note that the comments above on Perceptron and K2T image set apply here as well.

\subsection{Validation on real intensity images}

Recently, a large database of natural images with human segmentations has been made available for the research community [14]. The images were chosen from the Corel image database such that at least one discernable object is visible. Each image was segmented by several people. In doing so, quite different segmentations arise because either (I) the scene is perceived differently, or (II) the segmentation is done at different granularities; see Figure 4 for four example images with four segmentations each. In [14], the authors argue that if two different segmentations are caused by different perceptual organizations of the scene, then it is fair to declare the segmentations inconsistent. If, however, one segmentation is simply a refinement of the other, then the error should be small or even zero. Accordingly, they proposed the measures GCE and LCE discussed in Section 2. Due to their tolerance of refinement, a cluster $C_{1}$ containing a single cluster and a cluster $C_{2}$ consisting of clusters of a single object are rated by $\mathrm{GCE}=\mathrm{LCE}=0$. These two measures were used to conduct experiments by comparing all pairs of segmentations of the database (consisting of 50 images at that time). It was intended to show that despite the arguably ambiguous 
TABLE 2: Average index values for thirty ABW test images.

\begin{tabular}{ccccccccccc}
\hline Distance measure & $d=5 \%$ & $10 \%$ & $15 \%$ & $20 \%$ & $25 \%$ & $30 \%$ & $35 \%$ & $40 \%$ & $45 \%$ & $50 \%$ \\
\hline $\mathcal{R}$ & 0.024 & 0.041 & 0.055 & 0.068 & 0.080 & 0.091 & 0.102 & 0.111 & 0.120 & 0.129 \\
$\mathcal{F}$ & 0.046 & 0.079 & 0.105 & 0.129 & 0.152 & 0.171 & 0.190 & 0.206 & 0.221 & 0.235 \\
$\mathcal{I}$ & 0.088 & 0.146 & 0.191 & 0.229 & 0.264 & 0.293 & 0.320 & 0.343 & 0.364 & 0.382 \\
$\mathcal{D}$ & 0.027 & 0.046 & 0.063 & 0.078 & 0.092 & 0.105 & 0.117 & 0.128 & 0.138 & 0.149 \\
$\mathcal{B} \mathcal{G} \mathcal{M}$ & 0.027 & 0.047 & 0.064 & 0.079 & 0.094 & 0.108 & 0.121 & 0.133 & 0.144 & 0.155 \\
$\mathcal{N} \mathcal{M} \mathcal{l}$ & 0.725 & 0.740 & 0.751 & 0.761 & 0.770 & 0.777 & 0.784 & 0.790 & 0.796 & 0.801 \\
$\mathcal{V} I$ & 0.392 & 0.601 & 0.758 & 0.888 & 1.002 & 1.099 & 1.186 & 1.260 & 1.329 & 1.390 \\
\hline
\end{tabular}

TABLE 3: Index values for thirty ABW test images.

\begin{tabular}{lcccccccc}
\hline Algorithms & $\mathcal{R}$ & $\mathcal{F}$ & $\mathcal{g}$ & $\mathscr{D}$ & $\mathcal{B}$. $\mathcal{M}$ & $\mathcal{N} M I$ & $\mathcal{V} I$ & Hoover \\
\hline UE & 0.005 & 0.010 & 0.020 & 0.009 & 0.010 & 0.707 & 0.147 \\
UB & 0.008 & 0.016 & 0.031 & 0.013 & 0.014 & 0.714 & 0.209 \\
USF & 0.008 & 0.017 & 0.033 & 0.015 & 0.016 & 0.711 & 0.224 \\
UW & 0.009 & 0.017 & 0.033 & 0.019 & 0.025 & 0.848 & 0.236 \\
\hline
\end{tabular}

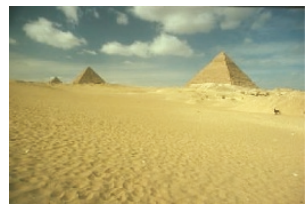

(a)

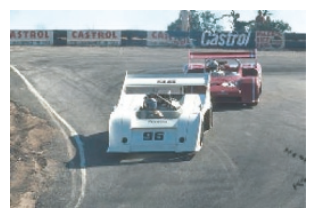

(f)

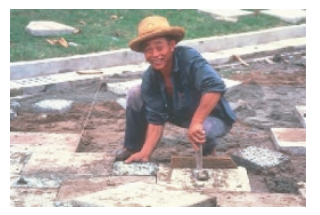

(k)

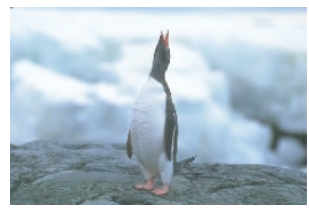

(p)

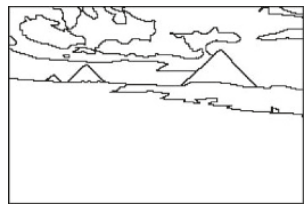

(b)

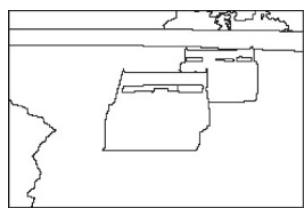

(g)

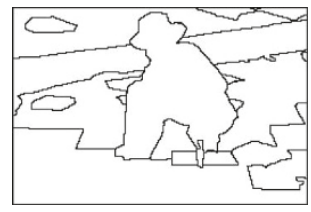

(1)

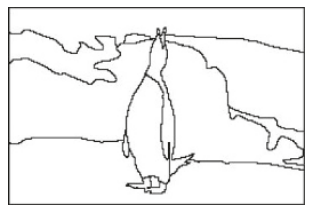

(q)

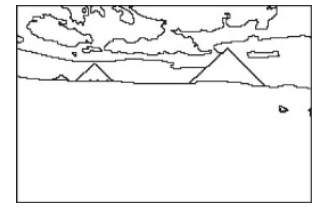

(c)

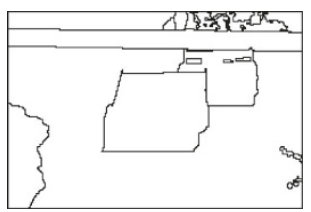

(h)

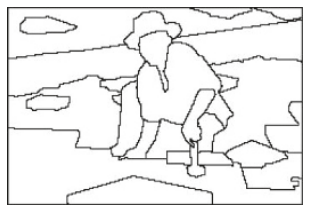

(m)

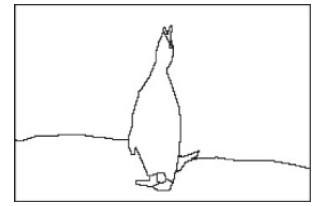

(r)

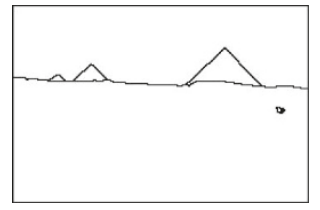

(d)

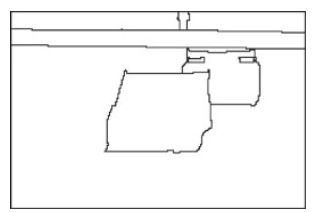

(i)

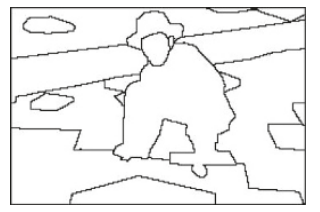

(n)

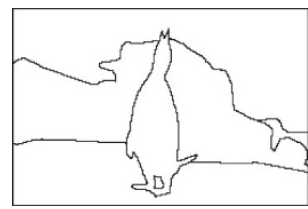

(s)

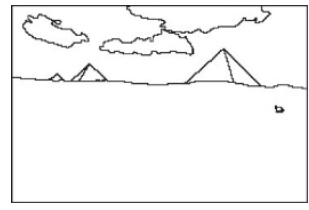

(e)

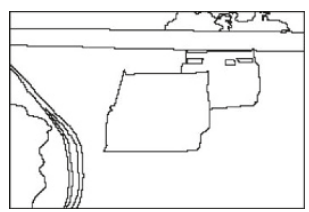

(j)

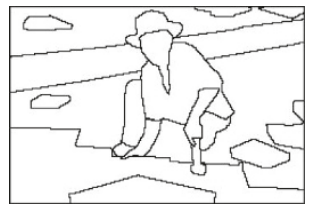

(o)

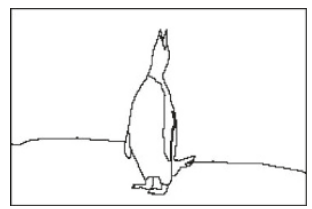

(t)

FIgURE 4: Example images from the database out of [14] and four human segmentations for each image. 
TABLE 4: Statistics of distance measures.

\begin{tabular}{lccccccccc}
\hline Error & $\mathcal{R}$ & $\mathcal{F}$ & $\mathscr{A}$ & $\mathscr{D}$ & $\mathcal{B} \mathscr{A} \mathcal{M}$ & $\mathcal{N} M I$ & $\mathcal{V} I$ & GCE & LCE \\
\hline$I_{\text {same }}$ & 0.117 & 0.197 & 0.317 & 0.123 & 0.215 & 0.772 & 1.114 & 0.087 & 0.055 \\
$I_{\text {diff }}$ & 0.378 & 0.622 & 0.792 & 0.446 & 0.645 & 0.943 & 3.424 & 0.441 & 0.375 \\
$\alpha$-error (\%) & 10.91 & 9.53 & 10.31 & 5.00 & 13.13 & 17.19 & 7.34 & 2.20 & 2.86 \\
$\beta$-error (\%) & 3.18 & 8.98 & 7.51 & 4.57 & 3.92 & 8.49 & 6.04 & 10.94 & 7.34 \\
\hline
\end{tabular}

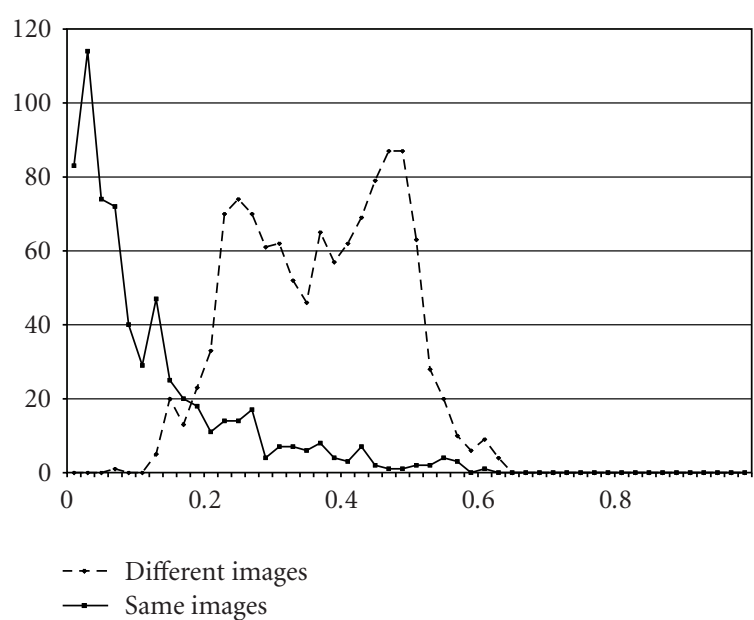

FIGURE 5: Distribution of Rand index.

nature of segmenting a natural image into an unspecified number of regions, different people produce consistent results on each image. In addition, the experiments help validating the measures by demonstrating that the distance between segmentations of the same image is low, while the distance between segmentations of different images is high.

We conducted a similar experiment to validate the measures proposed in this paper. For this purpose, 50 images were randomly selected from the database. Each of the images has at least five human segmentations. As an example, Figure 5 gives the distribution of the Rand index between pairs of human segmentations. As expected, the distance distribution for segmentations of the same image shows a strong spike near zero, while the distance distribution for segmentations of different images is neither localized nor close to zero. The average for all comparison cases of same images $I_{\text {same }}$ is 0.117 , while the average for different images amounts to $I_{\text {diff }}=0.378$. Obviously, the two distributions are not intersection-free, that is, using the Rand index, we will make some error in deciding whether two segmentations correspond to different segmentations of the same image (case (I)) or that of two different images (case (II)). This decision error can be quantified in the following way. We use the intersection point of the two curves as the decision threshold. Then, we call a decision case (II) made by the machine for the true case (I) an $\alpha$-error and a decision case (I) for the true case (II) an $\beta$-error. For the Rand index, the probability of $\alpha$-error and $\beta$-error is $10.91 \%$ and $3.19 \%$, respectively. The statistics for all the measures is listed in Table 4.
Obviously, they all tend to have large $\alpha$-error probability. The reason simply lies in the missing tolerance of segmentation refinement. Only the measure $\mathscr{D}\left(C_{1}, C_{2}\right)$ seems to have wellbalanced $\alpha$-error and $\beta$-error probabilities.

The behavior of the measure GCE and LCE from [14] is exactly converse. They tend to have small $\alpha$-error probability (due to the tolerance of refinement) and high $\beta$-error probability. It remains an interesting task to find measures with well-balanced $\alpha$-error and $\beta$-error probabilities (which are better than $\mathcal{D}\left(C_{1}, C_{2}\right)$ ).

\section{CONCLUSIONS}

Considering image segmentation as a task of data clustering opens the door for a variety of measures which are not known/popular in image processing. In this paper, we have presented several indices developed in the statistics and machine learning community. Some of them are even metrics. Experimental results have demonstrated their usefulness in both range image and intensity image domain. In fact, the proposed approach is applicable in any task of segmentation performance evaluation. This includes different imaging modalities (intensity, range, etc.) and different segmentation-tasks (surface patches in range images, texture regions in grey-level or color images). In addition, the usefulness of these measures is not limited to evaluating different segmentation algorithms. They can also be applied to train the parameters of a single segmentation algorithm [10, 24].

Given some reasonable performance measures, we are faced with the problem of choosing a particular one in an evaluation task. Here it is important to realize that the performance measures may be themselves biased in certain situations. Instead of using a single measure, we may take a collection of measures and define an overall performance measure. One way of doing this could be to select one representative performance measure from each class of (similar) measures and to build an overall performance measure, for instance, by a linear combination. As a matter of fact, such a combination approach has not received much attention in the literature so far. We believe that it will achieve a better behavior by avoiding the bias of the individual measures. The performance measures presented in this paper provide candidates for this combination approach.

\section{APPENDIX}

Given the confusion matrix of size $k \times l$ and the notation $m_{i j}=\left|c_{i} \cap c_{j}\right|, c_{i} \in C_{1}, c_{j} \in C_{2}$, we derive the formulas for $N_{11}, N_{00}, N_{10}$, and $N_{01}$ as given in Section 3.4. 
From the definition, it immediately follows that

$$
\begin{aligned}
N_{11} & =\sum_{i=1}^{k} \sum_{j=1}^{l} \frac{m_{i j}\left(m_{i j}-1\right)}{2} \\
& =\frac{1}{2}\left[\sum_{i=1}^{k} \sum_{j=1}^{l} m_{i j}^{2}-\sum_{i=1}^{k} \sum_{j=1}^{l} m_{i j}\right] \\
& =\frac{1}{2}\left(\sum_{i=1}^{k} \sum_{j=1}^{l} m_{i j}^{2}-n\right) .
\end{aligned}
$$

In addition, we have

$$
\begin{aligned}
N_{10} & =\sum_{i=1}^{k}\left[\frac{n_{i}\left(n_{i}-1\right)}{2}-\sum_{j=1}^{l} \frac{m_{i j}\left(m_{i j}-1\right)}{2}\right] \\
& =\frac{1}{2}\left[\sum_{i=1}^{k} n_{i}^{2}-n\right]-\frac{1}{2}\left[\sum_{i=1}^{k} \sum_{j=1}^{l} m_{i j}^{2}-n\right] \\
& =\frac{1}{2}\left[\sum_{i=1}^{k} n_{i}^{2}-\sum_{i=1}^{k} \sum_{j=1}^{l} m_{i j}^{2}\right] .
\end{aligned}
$$

Analogously, it holds that

$$
N_{01}=\frac{1}{2}\left[\sum_{j=1}^{l} n_{j}^{2}-\sum_{i=1}^{k} \sum_{j=1}^{l} m_{i j}^{2}\right] .
$$

Finally,

$$
\begin{aligned}
N_{00} & =\frac{n(n-1)}{2}-N_{11}-N_{10}-N_{01} \\
& =\frac{1}{2}\left[n^{2}-\sum_{i=1}^{k} n_{i}^{2}-\sum_{j=1}^{l} n_{j}^{2}+\sum_{i=1}^{k} \sum_{j=1}^{l} m_{i j}^{2}\right] .
\end{aligned}
$$

\section{ACKNOWLEDGMENT}

The authors want to thank the maintainers of the Berkeley segmentation data set and benchmark for public availability.

\section{REFERENCES}

[1] X. Jiang, "Performance evaluation of image segmentation algorithms," in Handbook of Pattern Recognition and Computer Vision, C. H. Chen and P. S. P. Wang, Eds., pp. 525-542, World Scientific, Singapore, 3rd edition, 2005.

[2] X. Jiang and D. Mojon, "Supervised evaluation methodology for curvilinear structure detection algorithms," in Proceedings of 16th International Conference on Pattern Recognition (ICPR '02), vol. 1, pp. 103-106, Quebec, Canada, August 2002.

[3] M. S. Prieto and A. R. Allen, "A similarity metric for edge images," IEEE Transactions on Pattern Analysis and Machine Intelligence, vol. 25, no. 10, pp. 1265-1273, 2003.
[4] M. Sezgin and B. Sankur, "Survey over image thresholding techniques and quantitative performance evaluation," Journal of Electronic Imaging, vol. 13, no. 1, pp. 146-165, 2004.

[5] X. Jiang, C. Marti, C. Irniger, and H. Bunke, "Image segmentation evaluation by techniques of comparing clusterings," in Proceedings of 13th International Conference on Image Analysis and Processing (ICIAP '05), Cagliari, Italy, September 2005.

[6] A. Hoover, G. Jean-Baptiste, X. Jiang, et al., "An experimental comparison of range image segmentation algorithms," IEEE Transactions on Pattern Analysis and Machine Intelligence, vol. 18, no. 7, pp. 673-689, 1996.

[7] K. I. Chang, K. W. Bowyer, and M. Sivagurunath, "Evaluation of texture segmentation algorithms," in Proceedings of IEEE Computer Society Conference on Computer Vision and Pattern Recognition (CVPR '99), vol. 1, pp. 294-299, Fort Collins, Colo, USA, June 1999.

[8] X. Jiang, "An adaptive contour closure algorithm and its experimental evaluation," IEEE Transactions on Pattern Analysis and Machine Intelligence, vol. 22, no. 11, pp. 1252-1265, 2000.

[9] X. Jiang, K. W. Bowyer, Y. Morioka, et al., "Some further results of experimental comparison of range image segmentation algorithms," in Proceedings of 15th International Conference on Pattern Recognition (ICPR '00), vol. 4, pp. 877-881, Barcelona, Spain, September 2000.

[10] J. Min, M. W. Powell, and K. W. Bowyer, "Automated performance evaluation of range image segmentation algorithms," IEEE Transactions on Systems, Man and Cybernetics-Part B: Cybernetics, vol. 34, no. 1, pp. 263-271, 2004.

[11] M. W. Powell, K. W. Bowyer, X. Jiang, and H. Bunke, "Comparing curved-surface range image segmenters," in Proceedings of 6th IEEE International Conference on Computer Vision (ICCV'98), pp. 286-291, Bombay, India, January 1998.

[12] Q. Huang and B. Dom, "Quantitative methods of evaluating image segmentation," in Proceedings of International Conference on Image Processing (ICIP '95), vol. 3, pp. 53-56, Washington, DC, USA, October 1995.

[13] J. Freixenet, X. Muñoz, D. Raba, J. Martí, and X. Cufí, "Yet another survey on image segmentation: region and boundary information integration," in Proceedings of 7th European Conference on Computer Vision-Part III (ECCV'02), pp. 408-422, Copenhagen, Denmark, May 2002.

[14] D. Martin, C. Fowlkes, D. Tal, and J. Malik, "A database of human segmented natural images and its application to evaluating segmentation algorithms and measuring ecological statistics," in Proceedings of 8th IEEE International Conference on Computer Vision (ICCV '01), vol. 2, pp. 416-423, Vancouver, BC, Canada, July 2001.

[15] A. K. Jain, M. N. Murty, and P. J. Flynn, "Data clustering: a review," ACM Computing Surveys (CSUR), vol. 31, no. 3, pp. 264-323, 1999.

[16] W. M. Rand, "Objective criteria for the evaluation of clustering methods," Journal of the American Statistical Association, vol. 66 , no. 336, pp. 846-850, 1971.

[17] E. B. Fowlkes and C. L. Mallows, "A Method for comparing two hierarchical clusterings," Journal of the American Statistical Association, vol. 78, no. 383, pp. 553-569, 1983.

[18] A. Ben-Hur, A. Elisseeff, and I. Guyon, "A stability based method for discovering structure in clustered data," in Proceedings of 7th Pacific Symposium on Biocomputing (PSB '02), vol. 7, pp. 6-17, Lihue, Hawaii, USA, January 2002.

[19] S. van Dongen, "Performance criteria for graph clustering and Markov cluster experiments," Tech. Rep. INS-R0012, 
Centrum voor Wiskunde en Informatica (CWI), Amsterdam, The Netherlands, 2000.

[20] S. Khuller and B. Raghavachari, "Advanced combinatorial algorithms," in Algorithms and Theory of Computation Handbook, M. J. Atallah, Ed., chapter 7, pp. 1-23, CRC Press, Boca Raton, Fla, USA, 1999.

[21] T. M. Cover and J. A. Thomas, Elements of Information Theory, John Wiley \& Sons, Chichester, UK, 1991.

[22] A. Strehl, J. Ghosh, and R. Mooney, "Impact of similarity measures on web-page clustering," in Proceedings of 17th National Conference on Artificial Intelligence: Workshop of Artificial Intelligence for Web Search (AAAI'00), pp. 58-64, Austin, Tex, USA, July 2000.

[23] M. Meila, "Comparing clusterings by the variation of information," in Proceedings of 16th Annual Conference on Computational Learning Theory and 7th Workshop on Kernel Machines (COLT/Kernel '03), pp. 173-187, Washington, DC, USA, August 2003.

[24] L. Cinque, S. Levialdi, G. Pignalberi, R. Cucchiara, and S. Martinz, "Optimal range segmentation parameters through genetic algorithms," in Proceedings of 15th International Conference on Pattern Recognition (ICPR '00), vol. 1, pp. 474-477, Barcelona, Spain, September 2000.

Xiaoyi Jiang studied computer science at Peking University, China, and received his Ph.D. and Venia Docendi (Habilitation) degrees in computer science from the University of Bern, Switzerland. After a two-year period as a Research Scientist at the Cantonal Hospital of St. Gallen, Switzerland, he became an Associate Professor at the Technical University of Berlin, Germany. Currently, he is a Full Professor of computer sci-

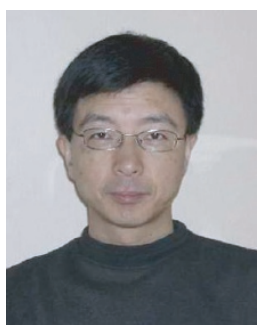

ence at the University of Münster, Germany. He is the coauthor of the book "Three-Dimensional Computer Vision: Acquisition and Analysis of Range Images" (in German), published by Springer and the Guest Coeditor of the Special Issue on Image/Video Indexing and Retrieval in Pattern Recognition Letters, April 2001. He was the coorganizer of the "Range Image Segmentation Contest" at the 15th International Conference on Pattern Recognition, Barcelona, 2000. Currently, he is the Editor-in-Charge of International Journal of Pattern Recognition and Artificial Intelligence. In addition, he is also serving on the editorial advisory board of International Journal of Neural Systems and the editorial board of the IEEE Transactions on Systems, Man, and Cybernetics-Part B, International Journal of Image and Graphics, and Electronic Letters on Computer Vision and Image Analysis. His research interests include multimedia databases, medical image analysis, vision-based man-machine interface, 3D image analysis, structural pattern recognition, and performance evaluation of vision algorithms.

Cyril Marti received the M.S. degree in computer science from the University of Bern, Switzerland. He is currently working as an Oracle Database Specialist at the Mimacom AG, Burgdorf. His research interests include pattern recognition and graph matching.
Christophe Irniger received the M.S. and Ph.D. degrees in computer science from the University of Bern, Switzerland. He is currently a Research Assistant with the Institute of Computer Science and Applied Mathematics at the University of Bern. His research interests include structural pattern recognition and data mining.

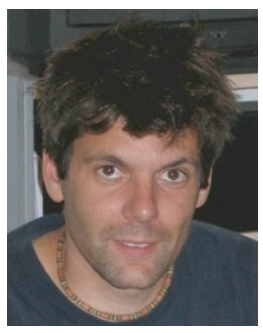

Horst Bunke received his M.S. and Ph.D. degrees in computer science from the University of Erlangen, Germany. In 1984, he joined the University of Bern, Switzerland, where he is a Professor in the Computer Science Department. From 1998 to 2000, he served as the first Vice-President of the International Association for Pattern Recognition (IAPR). In 2000, he also was the Acting President of this organization. He is a

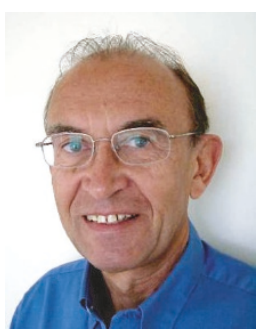
Fellow of the IAPR, former Editor-in-Charge of the International Journal of Pattern Recognition and Artificial Intelligence, Editorin-Chief of Electronic Letters of Computer Vision and Image Analysis, Editor-in-Chief of the book series on Machine Perception and Artificial Intelligence by World Scientific Publication Company, and the Associate Editor of Acta Cybernetica, the International Journal of Document Analysis and Recognition, and Pattern Analysis and Applications. He served as a Cochair of the 4th International Conference on Document Analysis and Recognition held in Ulm, Germany, 1997, and as a Track Cochair of the 16th and 17th International Conferences on Pattern Recognition held in Quebec City, Canada, and Cambridge, UK, in 2002 and 2004, respectively. He was on the program and organization committee of many other conferences and served as a referee for numerous journals and scientific organizations. He has more than 500 publications, including 33 authored, coauthored, edited, or coedited books and special editions of journals.

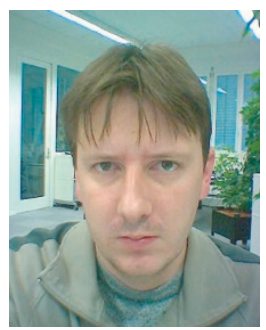

